\section{Serge Milbank}

is a digital specialist with

a career spanning 16 years

in Australia and the UK. He cofounded Stream:20 in 2005 in the belief that very soon digital would overtake offline channels as the main capture channel for sales. Stream:20 was established to help businesses get there through implementing robust systems and processes, working to establish digital sales culture. Stream:20 works with businesses such as Sky, More Than, Amazon, Betfair, Experian, EA, Sony and others to achieve these goals.

Keywords: digital marketing, benchmarking best practice, marginal gains, digital planning, strategy

Big gains based on little gains

\section{Business Intelligence}

\section{Marginal gains and innovating in digital marketing - A study on implementing best practice}

\author{
Serge Milbank \\ Received (in revised form): 28th January 2013
}

\begin{abstract}
The introduction of best practices inside organizations is akin to a series of marginal gains, that when aggregated together, will lead to significant gains in performance rather than any kind of great leap forward. Stream:20 introduce a benchmarking tool that allows businesses to assess where they sit in the implementation of best practice in a standardized scale-based assessment tool. Businesses can assess how they compare to a wider benchmark group. The Benchmarking tool - named the digital performance matrix organizes best practice implementation around four key pillars: (1) Digital Sales delivery, (2) Digital Sales Planning, (3) Digital Sales Toolkits and (4) Organizational Structure. Insights presented from the use of the benchmarking tool demonstrate that businesses tend to have a bias toward improving areas they are already strong at, leading to uneven development of capabilities. Businesses that are pushing ahead in terms of capabilities also tend to have strong planning functions within their organization. Readers are encouraged to assess their digital marketing function against benchmarks using the Digital Performance Matrix.

Journal of Direct, Data and Digital Marketing Practice (2013) 14, 249-256. doi:10.1057/dddmp.2013.16
\end{abstract}

\section{Background}

One of the stated goals of the London Olympics was to 'inspire a generation', and like many things inspirational, there was a range of unintended, but positive outcomes. This study was commissioned on the back of one of those unintended inspirations.

The most successful British team in the Olympics was undoubtedly GB Cycling. It won nine medals, of which seven were Gold. The next best country (Australia) won a paltry single Gold. It would be fair to say that Great British track cycling dominated the opposition. Many people, including myself, started to ask the question, 'how did they do that?'

Towards the end of the track cycling event, the Performance Director of British Cycling, Dave Brailsford - who is also the man 


\section{Like cycling, digital marketing is an arms race}

behind Team Sky, which won the 2012 Tour de France — was interviewed on BBC Breakfast TV on 8th July 2012. Brailsford coined the term 'aggregation of marginal gains' which, in his view, underpinned Team GB's performance. He said: 'The whole principle of marginal gains came from the idea that if you broke down everything that could impact on a cycling performance, absolutely everything you could think of, and then you improved every little thing by 1 per cent. When you clump it all together, you get quite a significant increase in performance'.

We then heard about how his cyclists washed their hands really well, slept on the same pillow every night and had every aspect of their lives improved (as well as their bikes). This really rang true - I could just imagine what you could do if you had the funding to sit down and think about every minute element that could be improved, getting access to all the best technical experts in their fields (from space and military technology downwards) to help you and then had the time to implement all of those effectively.

\section{Why marginal gains are important in digital marketing}

As a business, Stream:20 spends a lot of time going into well-funded and not so well-funded digital marketing operations and working with them to make the most of their budgets. More often than not, we find a mentality that goes something like this: 'We need an initiative that's going to have a significant impact on sales. We cannot focus on initiatives that have a small impact, we are looking for a big lever that we can pull to drive a large volume'. This approach is a sort of antimarginal gains.

Coupled with this, the digital marketing marketplace is actually a level playing field. Everyone has access to pretty much the same technology. Some big spenders might get access to certain tech a little earlier, but that's about it. As soon as something new comes out (and rarely it is one of those big levers), all good digital marketing functions move fairly quickly to replicate what is being tried.

Digital marketing all of a sudden looks a lot like professional track cycling. It is a matter of how well you implement things.

\section{Measurement of marginal gains in digital marketing}

Following the Olympics, Stream:20 was inspired to sit down and put the principle of marginal gains into practice. The principle is clear: if there are a number of elements that improve digital marketing even if they are small — when you aggregate them together, you can achieve significant overall performance improvement. It worked for GB Cycling and it should work in digital.

We then set about developing the Digital Performance Matrix. This draws from the theory of aggregated marginal gains insofar as, in digital, it is often very hard to make a large leap forward. It is much easier and more reasonable to do many small things better. 


\section{Four pillars of digital performance}

\section{4 core areas for innovation}

\section{Identifying where to plan for success}

This approach also reduces risk in pursuing innovation. Doing 20 things and failing at 5 still leaves 15 successes. Doing one thing that takes up all your resources creates an untenable risk if you fail at it.

The Digital Performance Matrix is an aggregation of 127 key factors that we have judged can improve digital performance. We have organized these core factors into four main groupings or pillars:

- Digital Sales Delivery - focuses on digital marketing channels and how the e-Commerce operation deals with traffic, conversion and user experience;

- Digital Sales Planning - reviews campaign processes and strategic initiatives;

- Digital Toolkits - reviews the implementation of key technologies and tools that can help you run effectively;

- Organizational Structure - how your team is structured and how you interface outside and inside this team.

Under each pillar, there are 24 areas where opportunities for best practice and digital innovation can be pursued:

- Digital Sales Delivery - 11 core areas;

- Digital Sales Planning - five core areas;

- Digital Toolkits - five core areas;

- Organizational Structure - three core areas.

Under these core areas, there are a further 127 factors that represent a series of marginal gains that digital businesses can focus on and improve.

\section{Scoring marginal gains}

Once developed, Stream:20 set about scoring organizations on how they perform. In each element, an organization is scored on a scale of 0 (not implemented) to 5 (pure innovation). The scoring matrix allows an online marketing team to score itself on key elements that represent the best opportunity for success in digital. This can be aggregated up into key areas of operation, such as email marketing, paid search, reporting or technology implementation.

A digital marketing team then has an opportunity to make statements such as: 'We are scoring low on email marketing, behind competitors. We can improve ourselves by focusing on these three factors'. This provides a systematic means by which a business can plan for digital success.

\section{What does success look like?}

What has Stream:20 learned while reviewing online marketing operations? Probably, the key thing in this context is that best practice exists in pockets: organizations tend to be very good at some areas 


\section{Marketing is coping without good technology - Or a plan}

\section{Email suffers problems with data and IT}

(such as paid search, measurement and reporting), but not as good at others (display, social and Search Engine Optimization). It is very rare to find an organization that excels in all things digital.

We have been aggregating scores on the Digital Performance Matrix as it provides an opportunity to benchmark against the industry. The results provide an insight into what success looks like and how you can focus on improving your business. All data collected to date is aggregated to an overall score as to how well the benchmark group is rolling out best practice - this currently levels off at 2.6 out of 5 .

In other words, of all the activities that a business could do to implement best practice, at a broad level 52 per cent have been done. This leaves 48 per cent of opportunities for digital businesses to improve what they are doing. Such a low score should not scare you - it is designed to be very hard to get a high score. A low score merely represents an opportunity to grow.

The distribution of this figure is not even. When broken down by the four key pillars of best practice, variation starts to show. Looking at Sales Delivery (digital marketing channels and how an e-Commerce operation deals with traffic), the benchmark scores at 2.6, which matches the overall score. Looking at Sales Planning and Toolkits, the benchmark scores 2.4 , which is below the overall benchmark.

This number is revealing. It says key enablers for success (the ability to plan and the technology underpinning digital marketing) scoring 2.4 out of 5 are less well implemented than the marketing itself, which is 2.6 out of 5 . It seems marketers are doing the best they can without well-implemented technology and a plan that is limited in scope. Comparatively speaking, digital marketing teams are structured well, scoring 2.9 out of 5. Business is getting behind digital marketing, but opportunity knocks to enable these teams for greater success.

\section{Marginal gains: Drilling into delivery}

A score of 2.6 out of 5 really does not tell us much more than that there is an opportunity to improve in digital marketing. Luckily, this score is an aggregation of 71 areas of marginal gains, which allows for some good insight into how and where a digital marketing department can improve.

Figure 1 presents 41 of the 71 factors in Sales Delivery, specifically relating to inbound marketing. These represent areas of marginal gains that businesses can focus on to approach best practice. What is immediately evident from the graph is that there is a reasonable variation in how well developed some factors are compared with others.

As an example, let us examine a mature channel, such as email. Compared with the possibilities that email marketing presents, the benchmark group sits at 2.0, meaning that email is below average in terms of best practice. Drilling deeper into email, a fairly clear picture emerges: Reporting is relatively strong (score of 2.6) compared with Strategy (2.2) and Structure (2.3), which are below the average. Where the benchmark group falls behind is in the areas of Data, Targeting and 


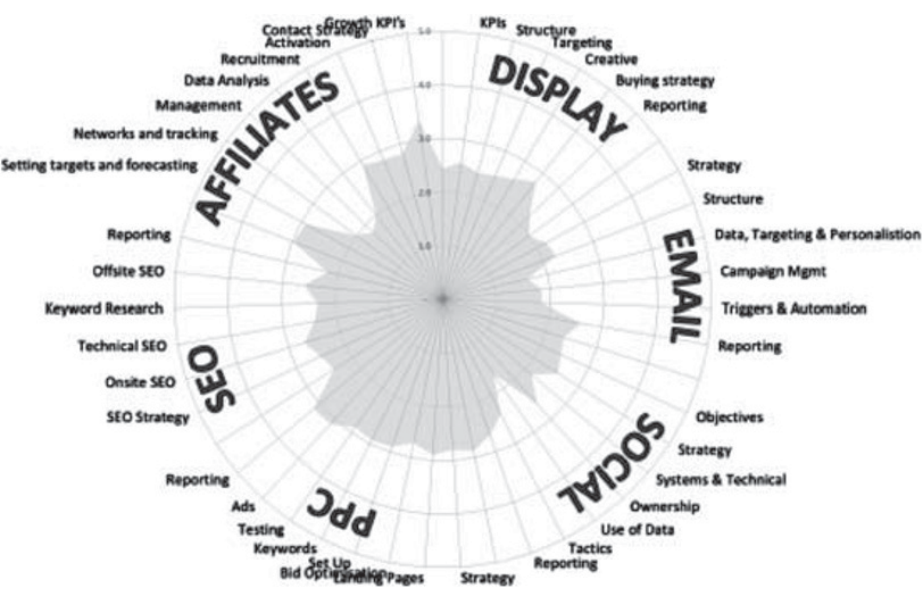

Figure 1: Benchmark and comparative scores for Sales Delivery (inbound marketing channels)

Pockets of excellence, areas of weakness

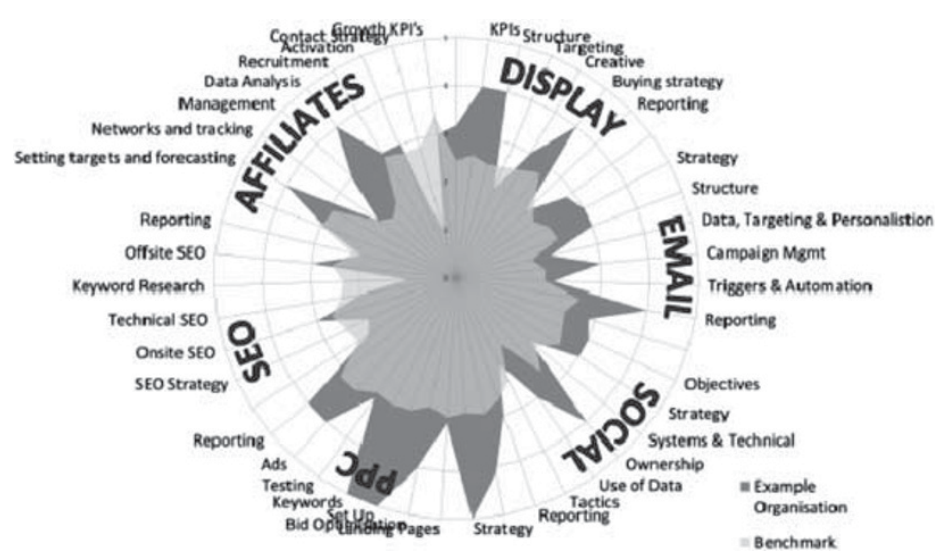

Figure 2: Benchmark versus example organization scores for Sales Delivery (inbound marketing channels)

Personalization (1.6), Campaign Management (1.8) and Triggers and Automation (1.8). These are low scores, representing areas where email marketing has consistently suffered from challenges because of IT and data constraints. These represent challenges on the client side that have not gone away.

\section{Comparing against the benchmark}

Figure 2 presents a different picture. It presents two sets of data - the benchmark group as in Figure 1, but with an overlay of data from an anonymous example organization. This example organization scores strongly on the Digital Performance Matrix with a score of 3.4, which represents a strong organization that is certainly approaching the limits of best practice. What is immediately evident is that the example organization is not pushing these limits in all areas — rather there are pockets of success, as well as areas where they are behind the benchmark. This is a consistent finding in the Digital Performance Matrix: best practice sits in pockets, rather than organization-wide. 


\section{Risk of getting better only at what you are already good at}

\section{What can we do to win?}

Using the same channel - email marketing - the example organization scores 2.8, ahead of the benchmark, but not streaks ahead in terms of email marketing. The example organization scores 4.0 on Reporting, well ahead of the benchmark score of 2.6. It is well ahead in all measures, but significantly stronger in Strategy (3.0), Structure (3.0) and Campaign Management (3.0) on the graph. These are clear factors that the example organization pushes ahead on. In the more data-heavy world of Data, Targeting and Personalization (2.0) and Triggers and Automation (2.0), the example organization is closer to the benchmark. If they are able to overcome these data issues, email should really progress into an area that pushes limits.

The important takeaway is that the example organization is not pushing limits in all areas. Rather it has pockets of brilliance, but is beset by the same issues all organizations have in making best practice happen. Importantly, progress is achieved in a series of marginal gains that in turn empower other areas.

\section{The role of planning in best practice improvement}

As businesses grow and progress when it comes to digital best practice, they tend to improve the areas that they are already good at. It is as if those pockets of brilliance are in fact what they are: teams (pockets) that are good in themselves and as a result of that talent (brilliance) have the ability to improve because of that talent or environment or technology.

This plays quite handily into the notion that improving performance in digital marketing is more about marginal gains and less about big levers. What is really happening in the example above is that a few core areas improve marginally, which results in the appearance of a big lever being pulled. The big levers do not exist - rather they exist, but as an aggregation of marginal gains. The route to the big levers is therefore a series of marginal improvements in elements of best practice over time.

The challenge, of course, is to have businesses improve in areas they are not good at and this is not easy. In many of these instances, you cannot rely on teams of people that have the ability to improve as a result of talent, environment or technology. Those steps tend to be small and incremental and very rarely the great leap forward requested by management.

Therefore, faced with the reality that there are very few big wins that are quick and easy in the pursuit of winning against competitors, the question arises - what can we do? In keeping with the theme of Olympic legacy, we should turn once again to our hero Dave Brailsford, who answered this question very well when asked how we could have won so many Golds in the Olympics as well as the Tour De France. He said: 'So we really figure out what it would take to win whatever it is that we want to win. We then prioritize, because you know you can't win everything. You will lose more than you win, that's for sure. So we decided what we want to win and when and then we work back to where we are today and look at the gap between 


\section{Planning and success are clearly related}

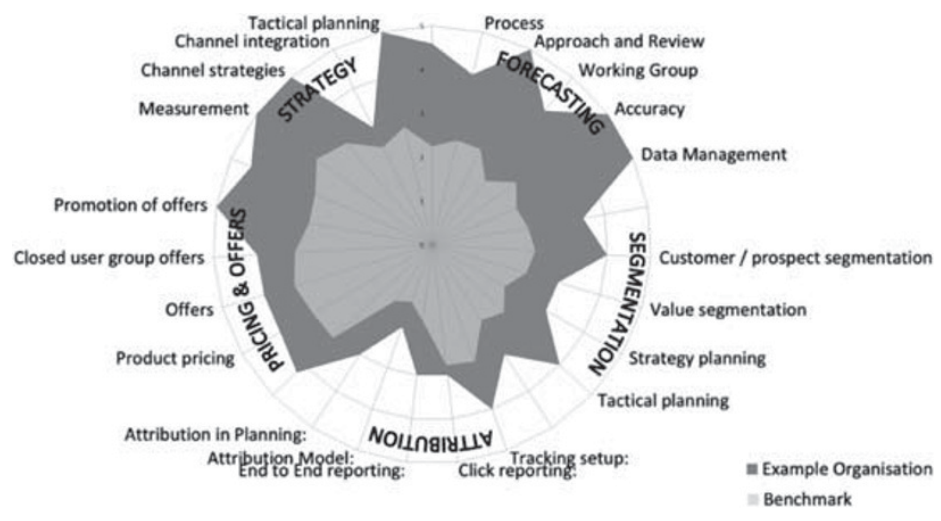

Figure 3: Benchmark versus example organization scores for Planning

where are today and what we want to win and create a plan and execute it'.

The above quote demonstrates a brilliant planner in full stride. Objectives were set, acknowledging that not everything can be won. However, the big ones that matter can be won if you focus on them and a systematic plan is put in place to achieve those specific objectives. The previous examples support this - digital marketing departments cannot be great at everything. They end up being great at some things and fall behind in others.

How can an online marketing department progress itself beyond its own pockets of brilliance? Enter the outlier - planning. There is a clear and obvious relationship between planning and eventual success. A business sits down, attaches a value to success in a few specific areas that are outside of its areas of expertise and sets out a specific plan to achieve that. This acknowledges the reality of finite resources - you cannot do everything. A business that plans well has a much higher probability of achieving success through marginal gains than those which do not.

Figure 3 outlines the Digital Performance Matrix benchmark results for the key area of Planning in digital marketing compared with our example organization. What is immediately clear here is that the example organization is ahead on all benchmarks when it relates to Planning. To labour a point, they are not equally innovative — some areas still have room for improvement.

In Forecasting, the example organization is beyond pushing limits its innovating (scoring 4.6 compared with a benchmark of 2.3). The example organization is achieving full scores of 5.0 out of 5.0 in Approach and Review, Accuracy and Data Management. It is pushing limits in Process and Planning Working Groups (each 4.0). These scores are well ahead of the benchmarks.

An important comparison now needs to be made between Planning and Sales Delivery. It is no mistake that the example organization is doing well in terms of Sales Delivery. The example organization is clearly progressing in key areas of Sales 
Finding how to achieve a step change in performance
Delivery - and it is down to Planning. You tend to find organizations progress once they have a strong planning function in place. As with Team GB track cycling, in a strong planning environment, objectives are set and opportunities or factors are identified, prioritized and resources are dedicated specifically to that priority. It provides the environment to allow success in areas that the business is not already good at.

\section{Summary}

The Digital Performance Matrix is a comprehensive means by which an organization can assess where it sits on the innovation continuum. The results of the tool provide a framework for deciding where to focus resources on improving digital marketing performance.

The conclusions presented in this paper remain general - in the context of implementing best practice digital marketing within an organization, marginal gains are important. Step changes tend to be made in increments and in an uneven way. Organizations have an inherent bias towards areas where they are already strong in the pursuit of best practice. This leads to uneven development of capabilities. In itself, this need not be a problem, but to achieve fundamental shifts in performance, areas that are lacking need focus outside of the usual biases of the organization.

The solution to improving performance lies in building up a strong planning function within the organization. This precedes performance improvement in Sales Delivery or marketing channels. The planning function allows prioritization of resources towards areas that the organization does not usually excel in, as well as providing an opportunity to quantify the prize.

How can you benefit further? The Digital Performance Matrix is progressively increasing the pool of companies within its sample group. We would encourage you to take the time to fill out the survey for yourself at www.stream20.com and match your business to the benchmark group. It will provide you with a framework for identifying where to focus energy in the short, medium and longer term. 